\title{
The New Role of CD163 in the Differentiation of Bone Marrow Stromal Cells into Vascular Endothelial-Like Cells
}

\author{
Wei Lu, ${ }^{1}$ Le Su, ${ }^{1}$ Zhezheng Yu, ${ }^{1}$ Shangli Zhang, ${ }^{1}$ and Junying Miao ${ }^{1,2}$ \\ ${ }^{1}$ Shandong Provincial Key Laboratory of Animal Cells and Developmental Biology, School of Life Science, Shandong University, Jinan \\ 250100, China \\ ${ }^{2}$ The Key Laboratory of Cardiovascular Remodeling and Function Research, Chinese Ministry of Education and Chinese Ministry of \\ Health, Shandong University Qilu Hospital, Jinan 250012, China
}

Correspondence should be addressed to Junying Miao; miaojy@sdu.edu.cn

Received 15 August 2015; Revised 29 October 2015; Accepted 3 November 2015

Academic Editor: Laura Lasagni

Copyright (C) 2016 Wei Lu et al. This is an open access article distributed under the Creative Commons Attribution License, which permits unrestricted use, distribution, and reproduction in any medium, provided the original work is properly cited.

\begin{abstract}
Bone marrow stromal cells (BMSCs) can differentiate into vascular endothelial cells (VECs). It is regarded as an important solution to cure many diseases, such as ischemic diseases and diabetes. However, the mechanisms underlying BMSC differentiation into VECs are not well understood. Recent reports showed that CD163 expression was associated with angiogenesis. In this study, overexpression of CD163 in BMSCs elevated the protein level of the endothelial-associated markers CD31, Flk-1, eNOS, and VEcadherin, significantly increased the proportion of Alexa Fluor 488-acetylated-LDL-positive VECs, and promoted angiogenesis on Matrigel. Furthermore, we demonstrated that CD163 acted downstream homeobox containing 1 (Hmboxl) and upstream fibroblast growth factor 2 (FGF-2). These data suggested that CD163 was involved in Hmbox1/CD163/FGF-2 signal pathway in BMSC differentiation into vascular endothelial-like cells. We found a new signal pathway and a novel target for further investigating the gene control of BMSC differentiation into a VEC lineage.
\end{abstract}

\section{Introduction}

Bone marrow stromal cells (BMSCs), due to their low immunogenicity and multilineage differentiation potential, promise as a main actor of cell-based therapeutic strategies [1]. They are able to differentiate into various kinds of cells, including vascular endothelial cells (VECs), osteocytes, chondrocytes, adipocytes, and neural cells [2-4]. VECs have been shown to play a central role in vasculogenesis during development and angiogenesis [5]. After being implanted into vein grafting in rats, these BMSCs differentiate into VECs and inhibit advanced vascular lesion formation [6]. Therefore, BMSCs' transplantation is regarded as an important strategy to ameliorate much damage or cure diseases, including spinal cord injury, disc degeneration, cerebral ischemic disease, and diabetes [7]. However, the mechanism of BMSC differentiation into VECs is not well-known.

BMSCs can be induced to differentiate into VECs under certain conditions in vitro [2, 8-10]. (1) The classical proangiogenic inducer VEGF, FGF-2, and PDGF could turn on this differentiation through MAKP signaling pathway [8].
(2) Simvastatin enhances this process via notch signaling pathway [9]. (3) Our previous study showed that chemical small molecule, 6-amino-2,3-dihydro-3-hydroxymethyl-1,4benzoxazine $(\mathrm{ABO})$, could promote $\mathrm{BMSC} /$ mouse embryonic stem cell (mESC) differentiation into VECs by elevating homeobox containing 1 (Hmboxl) expression $[2,10]$.

CD163 was first identified in 1987 and received its CD number in 1996 [11, 12]. It was widely thought that expression of CD163 is restricted to cells of the monocyte/macrophage lineage $[13,14]$. However, recent analyses have revealed that the tumor cell itself in breast, rectal, and bladder cancer expressed CD163 [15]. Moreover, CD163 is also highly expressed in endothelial progenitor cells (EPCs) [16]. Since EPCs have been shown to be involved in neovascularization and angiogenesis, it is suggested that CD163 may be associated with endothelial differentiation.

CD163 belongs to the cysteine-rich scavenger receptor superfamily type B [11]. Most studies focus on inflammation. In monocyte/macrophage lineage, expression of CD163 is tightly regulated, with a general tendency of 


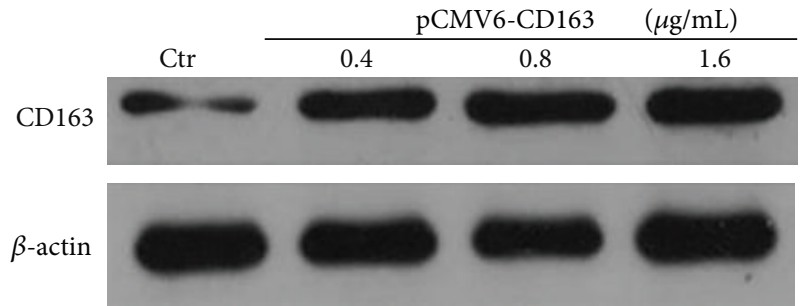

(a)

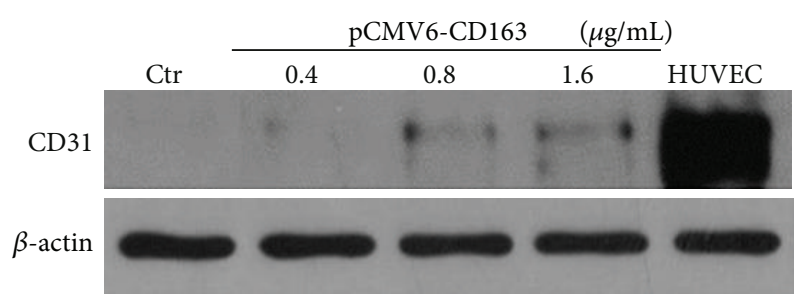

(c)
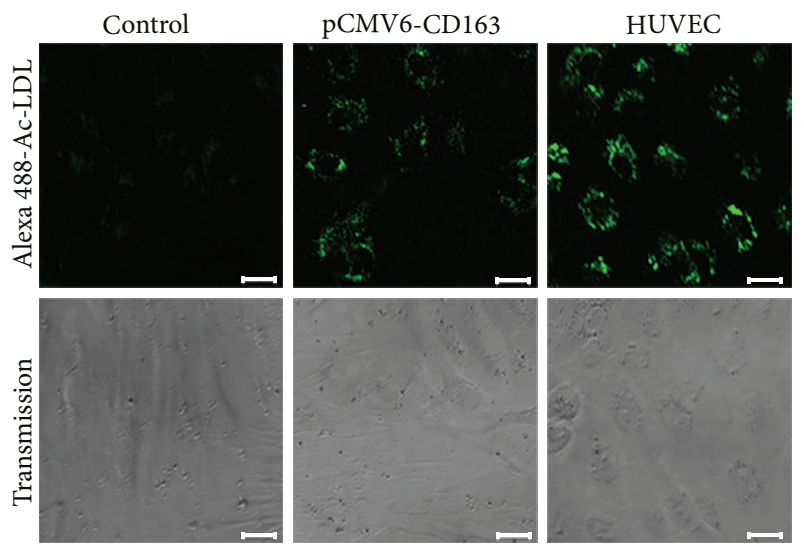

(e)

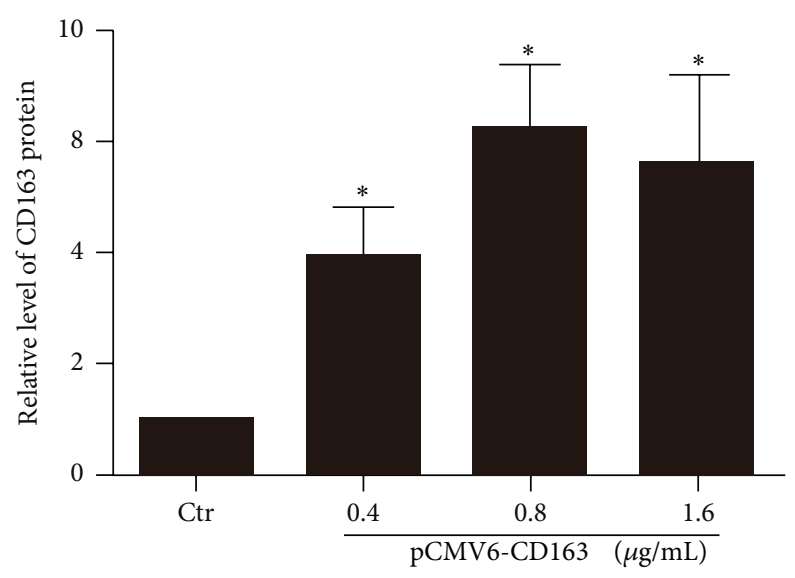

(b)

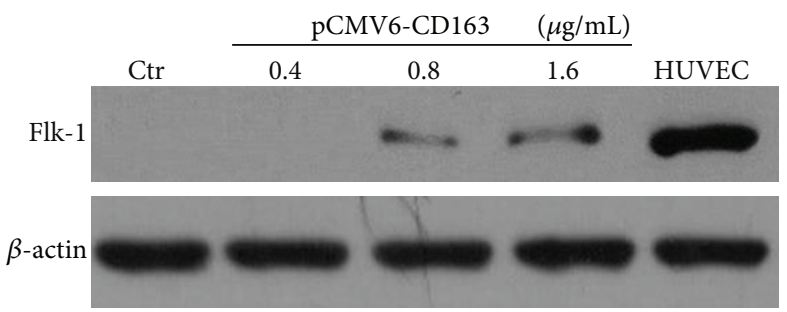

(d)

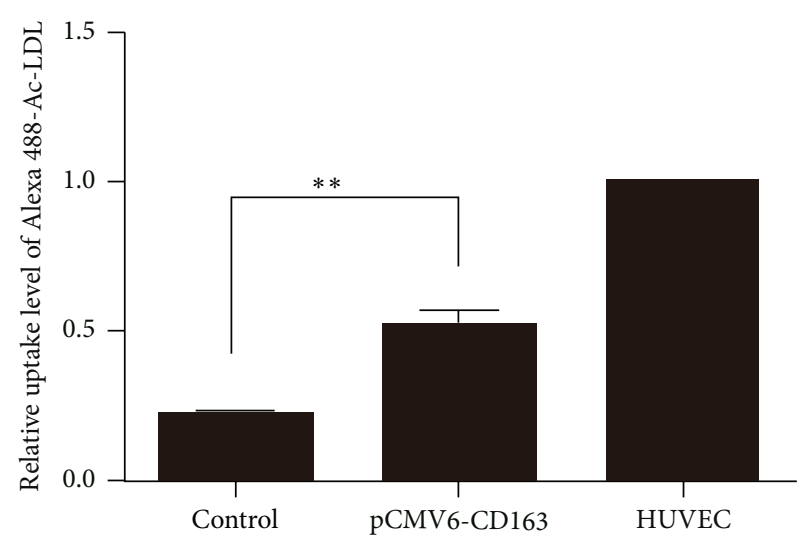

(f)

FIGURE 1: Overexpression of CD163 enhanced the expression of endothelial-associated markers and uptake of Alexa 488-Ac-LDL in BMSCs. For CD163 overexpression, BMSCs were transfected with empty vector pCMV6 (as control) at $1.6 \mu \mathrm{g} / \mathrm{mL}$ or pCMV6-CD163 at $0.4,0.8$, or $1.6 \mu \mathrm{g} / \mathrm{mL}$ for $4 \mathrm{~h}$ and cultured for $48 \mathrm{~h}$. Ctr: control. (a) Western blot analysis of CD163 overexpression efficiency. (b) Relative levels of CD163 protein were calculated based on densitometry analysis. ((c)-(d)) Western blot analysis of the protein level of the endothelial-associated markers CD31 and Flk-1 after being transfected with pCMV6 or pCMV6-CD163 for 48 h. (e) Uptake of Alexa 488-Ac-LDL by cells after being transfected with pCMV6 or pCMV6-CD163 at $0.8 \mu \mathrm{g} / \mathrm{mL}$ for $48 \mathrm{~h}$. Scale bar: $10 \mu \mathrm{m}$. Images are representative of at least 3 independent experiments. (f) Relative uptake level of Alexa 488-Ac-LDL was calculated by LCS lite. The uptake level of HUVEC was set as 1. HUVEC: human umbilical vascular endothelial cells as a positive control. Data are mean \pm SEM. ${ }^{*} p<0.05,{ }^{* *} p<0.01$ versus control (Ctr), $n \geq 3$.

anti-inflammatory signals to induce CD163 synthesis, while proinflammatory signals rather seem to downregulate CD163 expression [17]. The first-identified and most-studied function of CD163 is related to its capacity to bind and internalize haemoglobin-haptoglobin $(\mathrm{HbHp})$ complexes $[17,18]$. Later on, its functional repertoire was expanded, with the identification of CD163 as a receptor for tumour necrosis factorlike weak inducer of apoptosis (TWEAK), which is a TNF superfamily member and mediates angiogenesis [19-22]. Furthermore, recent report showed that CD163 expression is associated with angiogenesis [23]. Therefore, although CD163 has been most extensively studied in immunoregulatory context, there are many clues suggesting the relationship between CD163 and endothelial differentiation.

In our previous study, we found that the level of Hmboxl was upregulated in the differentiation of BMSC/mESC into 


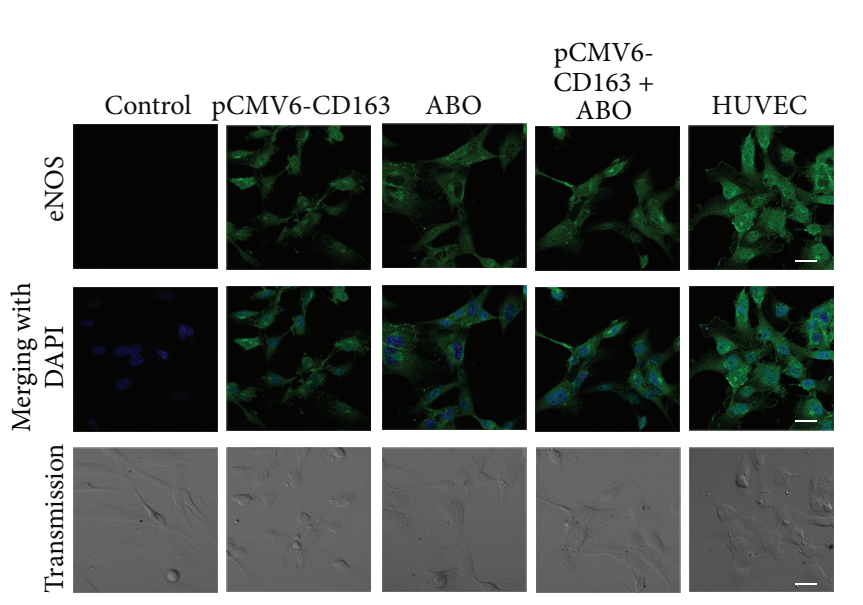

(a)

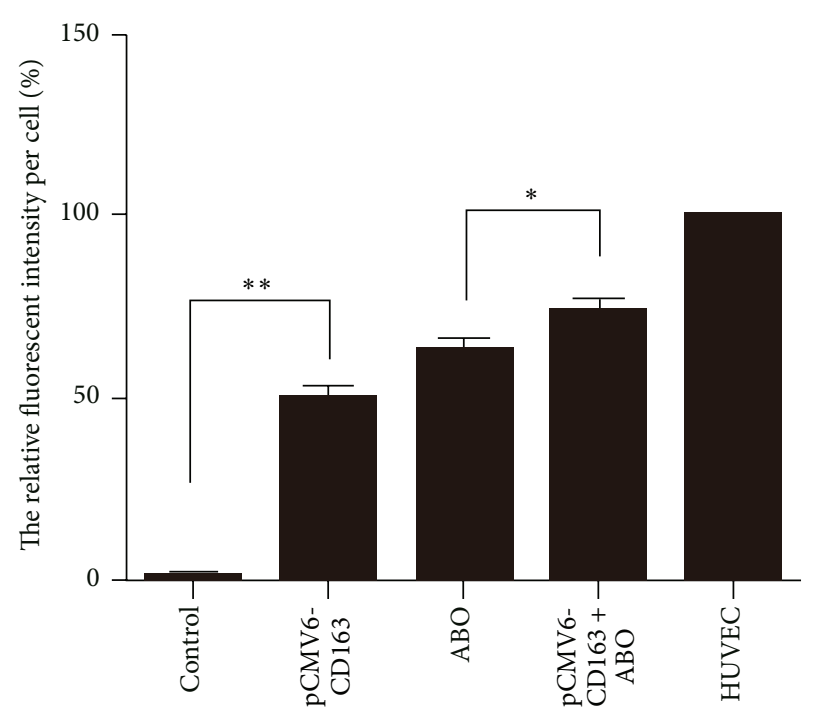

(b)

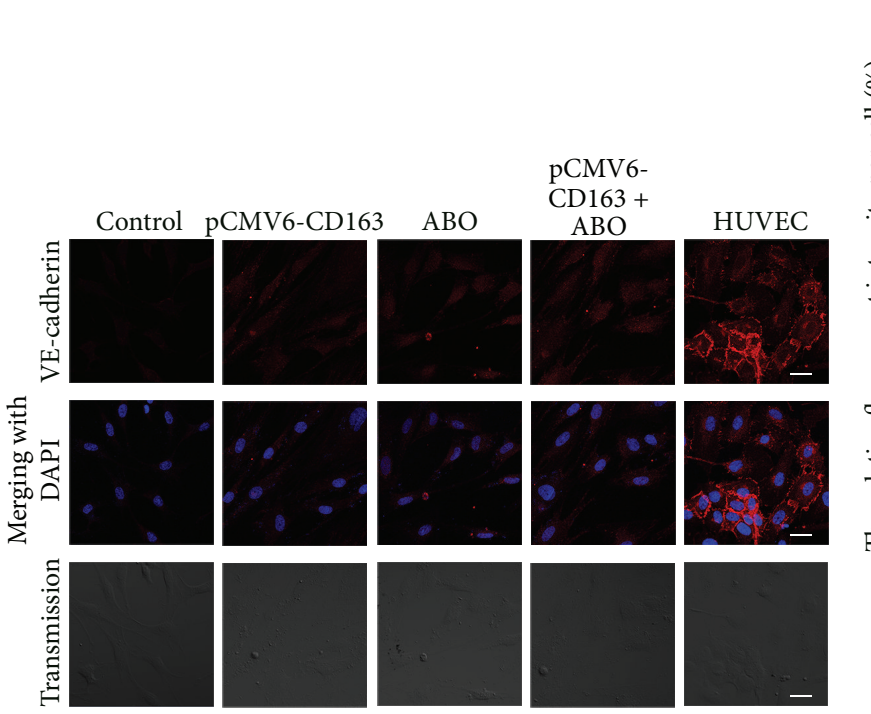

(c)

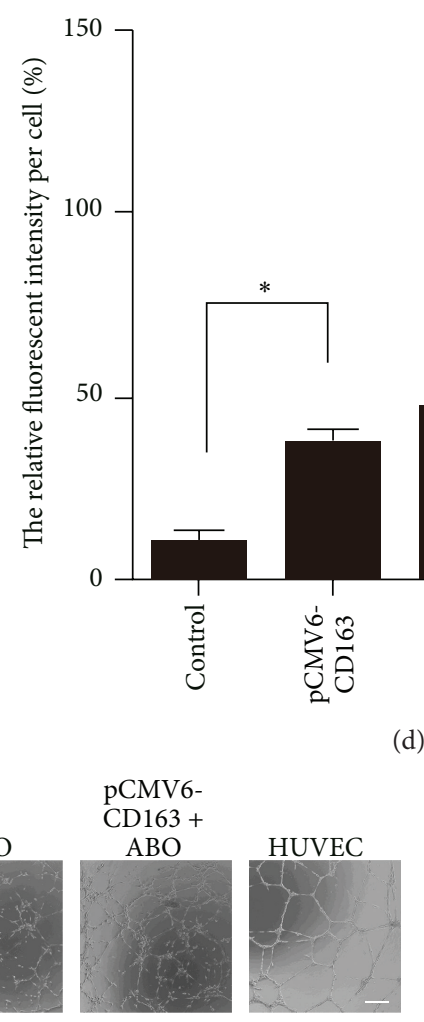

(e)

FIGURE 2: CD163 overexpression promoted BMSC differentiation into vascular endothelial-like cells with or without ABO treatment. In control group, BMSCs were transfected with empty vector pCMV6 and treated with $0.05 \%$ DMSO for 48 h; in pCMV6-CD163 group, BMSCs were transfected with pCMV6-CD163 for $48 \mathrm{~h}$; in ABO group, BMSCs were treated with $50 \mu \mathrm{M}$ ABO for $48 \mathrm{~h}$ (DMSO as control); in pCMV6$\mathrm{CD} 163+\mathrm{ABO}$ group, BMSCs were transfected with pCMV6-CD163 and treated with $50 \mu \mathrm{M}$ ABO for 48 h; and in HUVEC group, human umbilical vascular endothelial cells are considered as a positive control. (a) Immunostaining of the endothelial-function associated marker endothelial nitric oxide synthase (eNOS) in BMSCs treated as described above. Scale bar: $10 \mu \mathrm{m}$. (b) The relative fluorescent intensity was calculated and the fluorescent intensity of HUVECs was set as $100 \%$. (c) Immunostaining of the endothelial-specific marker VE-cadherin in BMSCs treated as described above. Scale bar: $10 \mu \mathrm{m}$. (d) The relative fluorescent intensity was calculated and the fluorescent intensity of HUVECs was set as $100 \%$. (e) The vascular structure formed on Matrigel by BMSCs treated as described above. Scale bar: $100 \mu \mathrm{m}$. Images are representatives of at least 3 independent experiments. Data are mean $\pm \mathrm{SEM} .{ }^{*} p<0.05,{ }^{* *} p<0.01, n \geq 3$. 


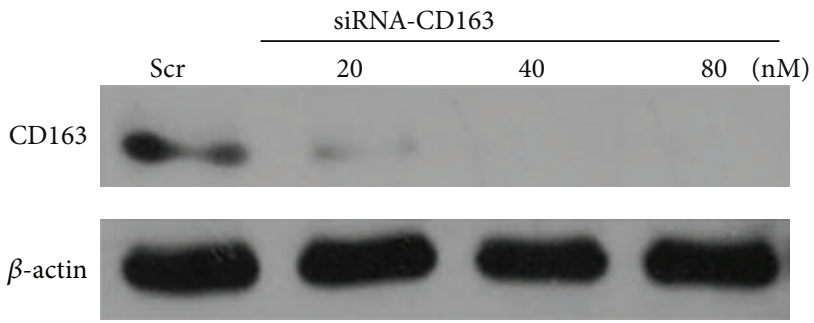

(a)

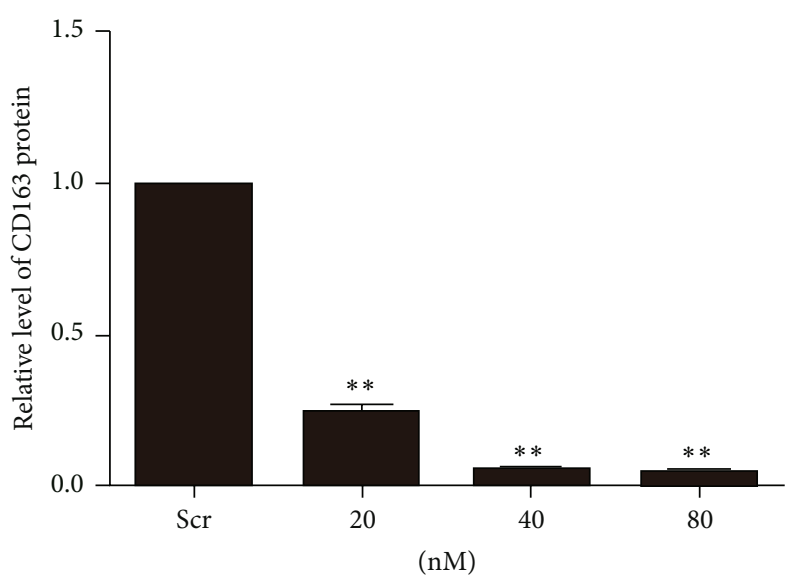

(b)

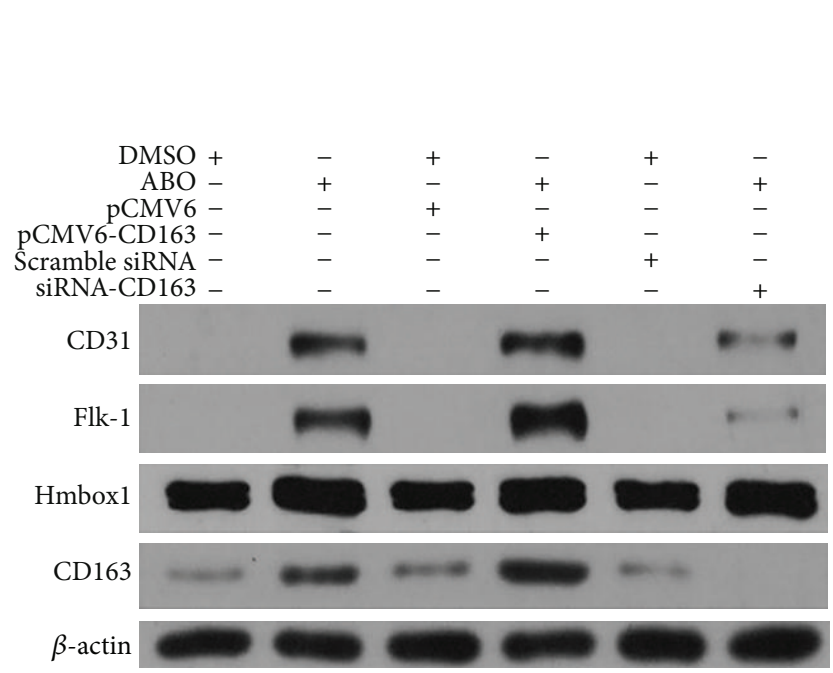

(c)

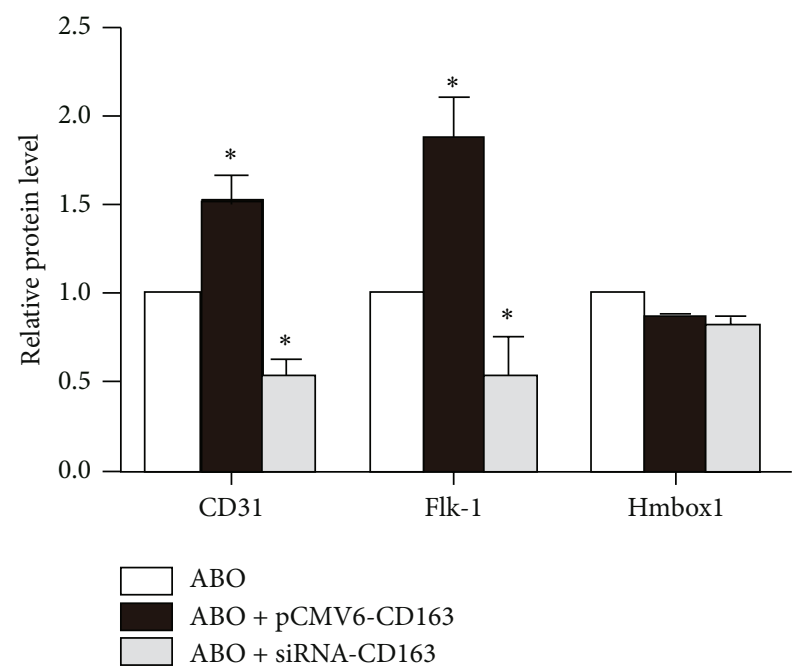

(d)

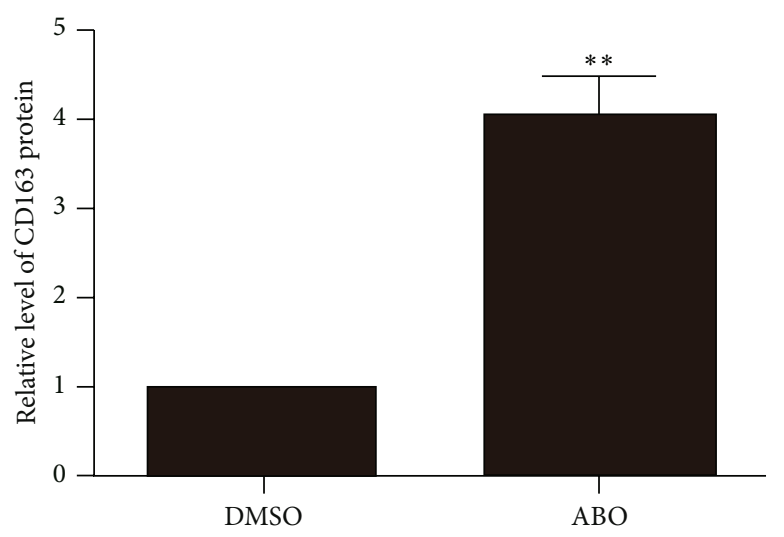

(e)

FIGURE 3: The protein level of CD163 influenced ABO-induced BMSC differentiation. For CD163 interference, cells were subjected with $80 \mathrm{nmol} / \mathrm{L}$ scrambled siRNA or 20, 40, and $80 \mathrm{nmol} / \mathrm{L}$ CD163 siRNA for $6 \mathrm{~h}$ and cultured for $48 \mathrm{~h}$. Scr: scrambled RNA as negative control. (a) Western blot analysis of CD163 RNA interference efficiency. (b) Relative levels of CD163 protein were calculated based on densitometry analysis. (c) Western blot analysis of the protein level of CD31, Flk-1, Hmboxl, and CD163 after overexpression or knockdown of CD163 with or without $50 \mu \mathrm{M}$ ABO treatment (DMSO as control). ((d)-(e)) Relative protein levels of CD31, Flk-1, Hmboxl, and CD163 were calculated based on densitometry analysis. Data are mean \pm SEM. ${ }^{*} p<0.05$ versus ABO group, ${ }^{* *} p<0.01$ versus control (Ctr), $n \geq 3$. 


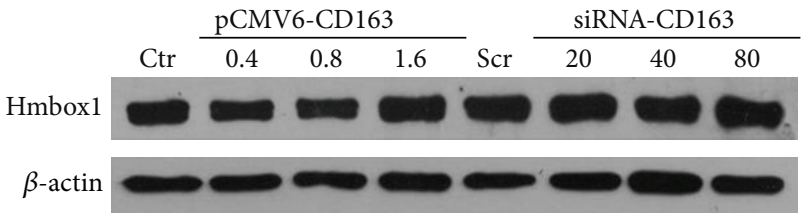

(a)

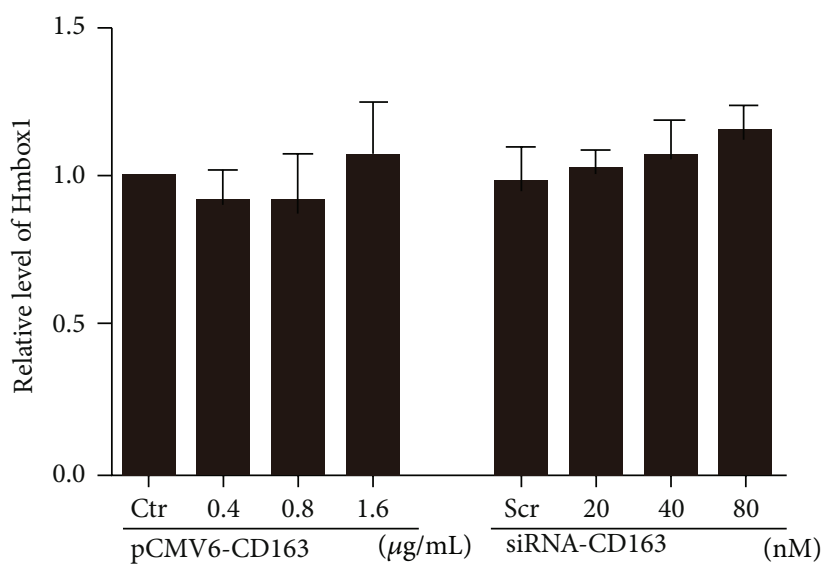

(b)

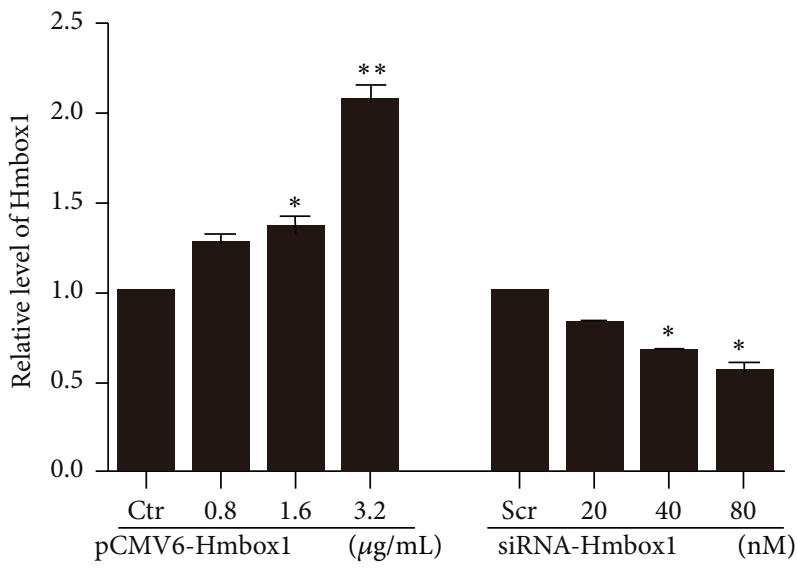

(d)

(c)

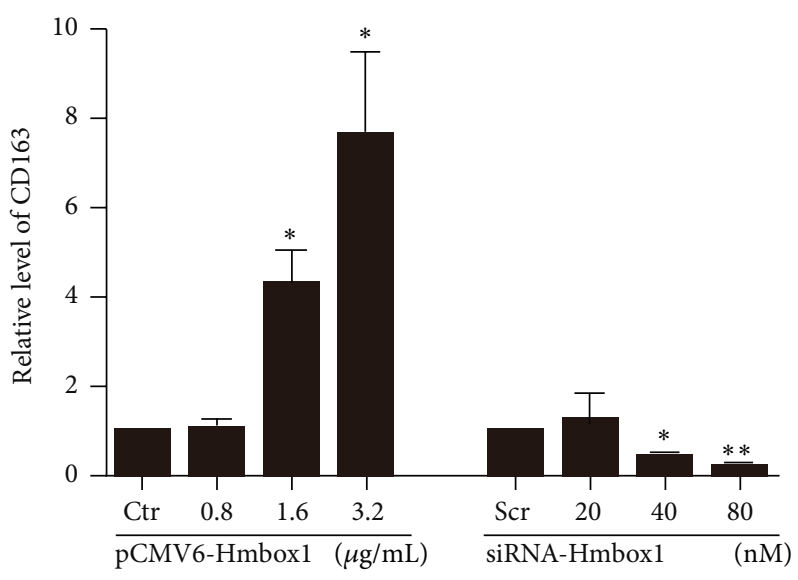

(e)

Figure 4: CD163 acted downstream Hmbox1 in differentiation of BMSCs towards a VEC lineage. (a) Western blot analysis of the protein level of Hmboxl after overexpression or knockdown of CD163. (b) Relative protein levels of Hmboxl were calculated based on densitometry analysis. (c) Western blot analysis of the protein level of Hmboxl and CD163 after overexpression or knockdown of Hmboxl. For Hmboxl overexpression, BMSCs were transfected with pCMV6 empty vector (as control) at $3.2 \mu \mathrm{g} / \mathrm{mL}$ or pCMV6-Hmboxl at $0.8,1.6$, or $3.2 \mu \mathrm{g} / \mathrm{mL}$ for $4 \mathrm{~h}$ and cultured for $48 \mathrm{~h}$. For Hmboxl interference, cells were subjected with $80 \mathrm{nmol} / \mathrm{L} \mathrm{scrambled} \mathrm{siRNA} \mathrm{or} \mathrm{20,} 40$, and $80 \mathrm{nmol} / \mathrm{L} \mathrm{Hmboxl}$ siRNA for $6 \mathrm{~h}$ and cultured for $48 \mathrm{~h}$. Scr: scrambled RNA as negative control. ((d)-(e)) Relative protein levels of Hmboxl and CD163 were calculated based on densitometry analysis. Data are mean \pm SEM. ${ }^{*} p<0.05,{ }^{* *} p<0.01$ versus control (Ctr), $n \geq 3$. 


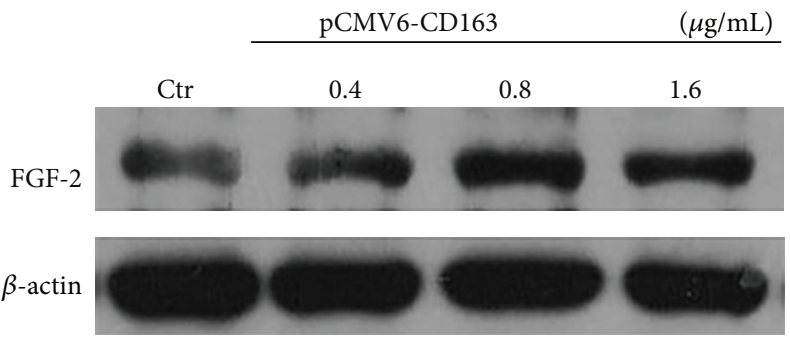

(a)

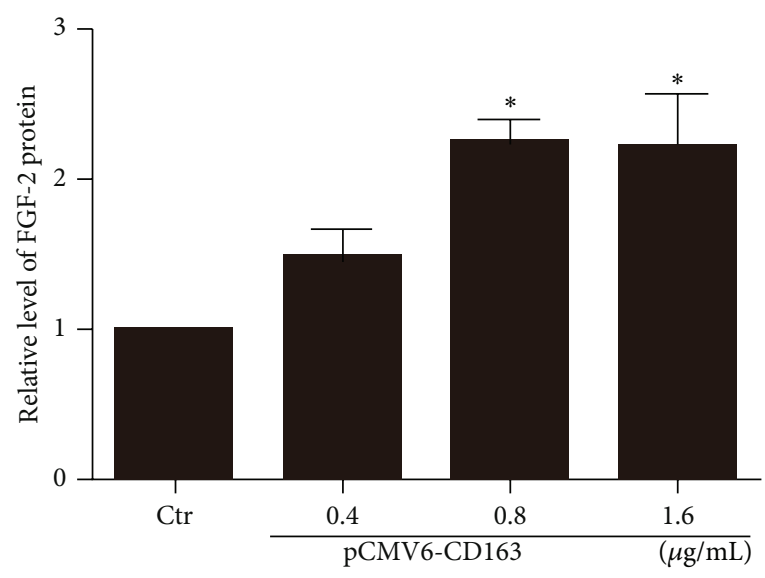

(b)

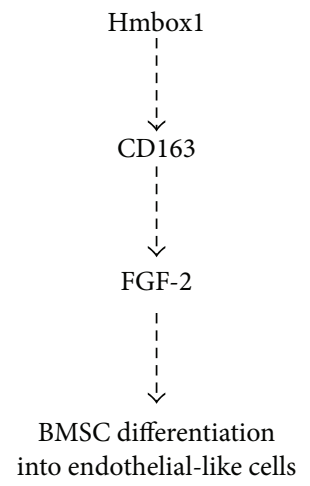

(c)

FIGURE 5: CD163 acted upstream FGF-2 in BMSC differentiation into a vascular endothelial lineage. (a) Western blot analysis of the protein level of FGF-2 after overexpression of CD163. (b) Relative protein levels of FGF-2 were calculated based on densitometry analysis. Data are mean \pm SEM. ${ }^{*} p<0.05,{ }^{* *} p<0.01$ versus control (Ctr), $n \geq 3$. (c) A conceptual schematic of CD163 involved in the differentiation of BMSCs into a vascular endothelial lineage. Hmboxl increases the expression of CD163, which promotes BMSC differentiation into vascular endothelial-like cells by enhancing FGF-2.

VECs induced by ABO. Moreover, ABO failed to induce the formation of VECs without Hmboxl $[2,10]$. Hence, Hmboxl was a key factor in BMSC/mESC differentiation into VECs. Furthermore, Hmboxl acted upstream the classical FGF-2 proangiogenic pathway in ABO-induced mESCs differentiation into VECs [10]. However, the relationship between CD163 and Hmbox1/FGF-2 signaling in BMSC differentiation into VECs was unknown. In this study, we demonstrated that CD163 was positively regulated by Hmboxl, and it could promote BMSC differentiation into vascular endothelial-like cells through enhancing FGF-2 signaling.

\section{Materials and Methods}

2.1. Cell Culture. Rat BMSCs were isolated from the femurs and tibias of male Wistar rats (90-100 g) as described earlier with modification [24]. Rats were killed by intravenous injection of ketamine/xylazine, and all work was performed according to the Institutional Animal Care and Use Committee guidelines. This experimental protocol was reviewed and approved by the Medical Ethics Committee of Shandong University School of Medicine. Briefly, the cells were seeded in Dulbecco's modified Eagle's medium low glucose (Gibco, USA) supplemented with 15\% fetal bovine serum and fibroblast growth factor 2 (FGF-2) (Beifuji, China), $10 \mathrm{ng} / \mathrm{mL}$, at $37^{\circ} \mathrm{C}$ in humidified air with $5 \% \mathrm{CO}_{2}$. Rat BMSCs were phenotypically characterized as described [25]. Cells were transfected with pCMV6 empty vector/pCMV6-CD163 in DMEM with FGF-2 (10 ng/mL), rVEGF (10 ng/mL) (Sigma, St. Louis, MO), and $2 \%$ serum and incubated at $37^{\circ} \mathrm{C}$ for $24 \mathrm{~h}$. Then, the medium is replaced by basal DMEM with FGF$2(10 \mathrm{ng} / \mathrm{mL})$ and $\mathrm{rVEGF}(10 \mathrm{ng} / \mathrm{mL})$ incubated at $37^{\circ} \mathrm{C}$ for another $24 \mathrm{~h}$.

2.2. Plasmids and Overexpression. BMSCs were cultured to $80 \%$ confluent prior to transfection in basal DMEM and transient transfection was performed using Lipofectamine 2000 (Invitrogen, 11668-019) transfection reagent according to the manufacturer's instructions. Cells were transfected with pCMV6-CD163 (Origene, RN206586), pCMV6Hmboxl (Origene, SC319800), or pCMV6 empty vector.

2.3. Alexa 488-Ac-LDL Uptake Assay. Cells were seeded on 24-well culture plates and incubated in $10 \mu \mathrm{g} / \mathrm{mL}$ Alexa 
Fluor 488-acetylated low-density lipoprotein (Alexa 488-Ac$\mathrm{LDL}$ ) (Invitrogen, USA) at $37^{\circ} \mathrm{C}$ for 4 to $6 \mathrm{~h}$. The medium was removed and cells were washed once with the culture medium. The cells that could uptake Alexa 488-Ac-LDL showed green fluorescence with LSCM excitation at $488 \mathrm{~nm}$. The ability to uptake Alexa 488-Ac-LDL was used to estimate the differentiation rate of BMSCs: the number of positively stained cells divided by the total number of cells in random visual fields. At least 200 cells for each sample were counted.

2.4. In Vitro Capillary-Like Tube Formation Assay. The formation of vascular-like structures in BMSCs was assessed by the use of Matrigel (YEASEN Biosciences, China) as previously described [26]. Briefly, cells were seeded on 24well plates coated with Matrigel at $4-5 * 10^{4}$ cells per well within DMEM with $15 \%$ serum and incubated at $37^{\circ} \mathrm{C}$ for 60 $90 \mathrm{~min}$. Then, cell culture medium was replaced by the basal DMEM. Finally, after 4-7 hours of initial cell seeding, tube formation was observed under an inverted-phase contrast microscope (Nikon, Japan).

2.5. RNA Interference. To knock down the expression of CD163 or Hmboxl, RNA interference (RNAi) was performed as described [27]. BMSCs were cultured to $50-60 \%$ confluence prior to transfection in basal DMEM, and transient transfection was performed using HiPerFect (Qiagen, 301704) transfection reagent according to the manufacturer's instructions. Cells were transfected with siRNA for CD163 (Origene, SR506300) or Hmboxl (designed and synthesized by Invitrogen, USA). The synthesized siRNA to Hmboxl was (RNA)-UUU CAG AGA CGU AAC UCG UUC CAG G (sense) and (RNA)-CCU GGA ACG AGU UAC GUC UCU GAA A (antisense). Scramble siRNA was used as a control (Santa Cruz Biotechnology, USA).

2.6. Immunofluorescence Assay. Immunofluorescence assay was performed as described previously [28]. After adding the primary antibodies, rabbit anti-eNOS IgG (Santa Cruz Biotechnology, sc-9989 1:100) or mouse anti-VE-cadherin IgG (Santa Cruz Biotechnology, sc-654 1:100), and appropriate secondary antibodies (fluorescein isothiocyanate- (FITC) - donkey anti-rabbit/mouse IgG; all Biotechnology, Santa Cruz, CA), the samples were evaluated by laser scanning confocal microscopy (LSCM) (Leica, Germany). eNOS displayed green fluorescence after excitation at $488 \mathrm{~nm}$ and VE-cadherin displayed red fluorescence after excitation at $546 \mathrm{~nm}$. We randomly selected the region of interest and then zoomed in the same frames. The relative fluorescent intensity (analyzed by ImageJ software) per cell was the total value of the sample in the zoom scan divided by the total number of cells (at least 200 cells) in the same scan.

2.7. Western Blot Analysis. Western blot was performed as described previously [29]. Briefly, the cells were lysed in protein lysis buffer containing $25 \mathrm{mM}$ Tris- $\mathrm{HCl}$ ( $\mathrm{pH} 6.8$ ), $2 \%$ SDS, 6\% glycerol, 1\% 2-mercaptoethanol, 2 mM PMSF, $0.2 \%$ bromophenol blue, and a protease inhibitor cocktail (Sigma, St. Louis, MO) for $10 \mathrm{~min}$ at RT (room temperature) and boiled for another $10 \mathrm{~min}$. The protein concentration was determined by Coomassie brilliant blue protein assay. The cellular proteins $(20 \sim 40 \mu \mathrm{g})$ were applied to $15 \%$ or $9 \%$ SDS polyacrylamide gel and electroblotted onto polyvinylidene difluoride (PVDF) membrane. The membrane was blocked with $5 \%(\mathrm{w} / \mathrm{v})$ nonfat dry milk in phosphate buffered saline (PBS), Tween 20 (PBST; 0.05\%) for $1 \mathrm{~h}$ and incubated with anti-CD163 (Santa Cruz Biotechnology, sc-58965 1:100), anti-Hmboxl (Santa Cruz Biotechnology, sc-87768 1:1000), anti-FGF-2 (Santa Cruz Biotechnology, sc-271847 1:1000), or anti- $\beta$-actin antibody (Santa Cruz Biotechnology, sc$477781: 1000)$ at $4^{\circ} \mathrm{C}$ overnight. After washing in PBST and PBS, the PVDF membrane was incubated with appropriate horseradish peroxidase-conjugated secondary antibodies (Santa Cruz Biotechnology 1:10000) for $1 \mathrm{~h}$ at RT. The membrane was incubated with Immobilon Western Chemiluminescent HRP Substrate (Millipore, WBKLS0500) for $5 \mathrm{~min}$ at RT and exposed to X-ray film (Kodak). The relative quantity of proteins was analyzed by the use of ImageJ software and normalized to loading controls.

2.8. Statistical Analyses. Data are expressed as mean \pm SEM. Images were processed by use of Adobe Photoshop CS4 (Adobe, San Jose, USA). SPSS 20.0.0 (SPSS Inc., Chicago, IL) was used for statistical analysis. Comparisons among groups involved one-way ANOVA followed by Scheffé $F$-test post hoc analysis. A $p<0.05$ was considered statistically significant.

\section{Results}

3.1. CD163 Promoted BMSC Differentiation into Vascular Endothelial-Like Cells. Since many studies suggested the relationship between CD163 and endothelial differentiation, we intend to investigate the role of CD163 in the differentiation of BMSCs to VECs. The full-length cDNA sequence of CD163 was cloned into the pCMV6 expression vector, pCMV6CD163. BMSCs were transfected with pCMV6 empty vector at $1.6 \mu \mathrm{g} / \mathrm{mL}$ or pCMV6-CD163 at $0.4,0.8$, and $1.6 \mu \mathrm{g} / \mathrm{mL}$ for $48 \mathrm{~h}$ with rVEGF and FGF-2. The efficiency of overexpression CD163 was confirmed by western blotting. Compared with pCMV6 empty vector, pCMV6-CD163 increased the protein level of CD163 in BMSCs in a dose-dependent manner (Figures 1(a) and 1(b)). To confirm the role of CD163 in the endothelial differentiation of BMSCs, we detected the protein level of the endothelial-associated markers CD31 and Flk-1 by western blotting after being transfected with pCMV6-CD163 or pCMV6 empty vector for $48 \mathrm{~h}$ in BMSCs. Human umbilical vein endothelial cells (HUVECs) were set as a VECs positive control. The results showed that protein levels of the endothelial-associated markers CD31 and Flk1 were undetectable in BMSCs transfected with pCMV6 empty vector, but they were observed in BMSCs transfected with pCMV6-CD163 at $0.8,1.6 \mu \mathrm{g} / \mathrm{mL}$ (Figures 1 (c) and 1(d)). It is suggested that CD163 promoted the expression of endothelial-associated markers CD31 and Flk-1 in BMSCs.

VECs are functionally defined by their uptake capacity to acetylated low-density lipoprotein from plasma. For 
further investigating the differentiation rate and defining the function of CD163 overexpression BMSC-derived VECs, we detected a number of Alexa Fluor 488-acetylated lowdensity lipoprotein-positive VECs in CD163 overexpression BMSCs. As shown in Figures 1(e) and 1(f), overexpression of CD163 significantly increased the number of Alexa Fluor 488acetylated-LDL-positive cells. HUVECs were set as a VEC positive control. The differentiation proportion of BMSCs was $52.6 \%$ after transfection with pCMV6-CD163, whereas the proportion of the control group was only $22 \%$. This result showed that CD163 overexpression in BMSCs effectively enhanced the uptake of Alexa Fluor 488-acetylated lowdensity lipoprotein (Alexa 488-Ac-LDL).

Furthermore, we also examined the expression of the endothelial-function associated marker endothelial nitric oxide synthase (eNOS) and the endothelial-specific marker VE-cadherin by immunofluorescence. eNOS is tightly associated with endothelial function and vascular homeostasis. Its expression was dramatically induced by CD163 overexpression in BMSCs (Figures 2(a) and 2(b)). Compared with the control group, the expression of the endothelial-specific marker VE-cadherin was induced after BMSC transfected with pCMV6-CD163 as well (Figures 2(c) and 2(d)). The data suggested that the overexpression of CD163 promoted the expression of endothelial-function associated marker eNOS and endothelial-specific marker VE-cadherin in BMSCs.

Angiogenesis is the process where new blood vessels sprout out of preexisting vessels and this process involves the recruitment and proliferation of VEC [30, 31]. In vitro angiogenesis assay has shown that mature VECs form a capillary-like network when plated on Matrigel [32]. We used a Matrigel based angiogenesis assay to define the functionality of the CD163 overexpression BMSCs derived vascular endothelial-like cells and HUVECs were set as a VEC positive control. Unlike the control group, capillary-like network was observed in BMSCs transfected with pCMV6CD163 and in HUVECs (Figure 2(e)).

Collectively, our data suggested that, compared with the control group, BMSCs with CD163 overexpression enhanced the differentiation of BMSCs into vascular endothelial-like cells.

3.2. CD163 Played an Important Role in ABO-Induced Differentiation of BMSCs to VECs. In our previous study, we found that $\mathrm{ABO}$ could induce BMSC differentiation into VECs by elevating Hmboxl expression [2]. We wondered whether CD163 participated in ABO-induced differentiation of BMSCs to VECs. And we found that, compared with $\mathrm{ABO}$ group, the angiogenesis capacity and the expression of eNOS and VE-cadherin were further enhanced by CD163 overexpression and $\mathrm{ABO}$ treatment (Figure 2). It suggested that CD163 might be involved in ABO-induced endothelial differentiation of BMSCs.

To verify whether CD163 participates in ABO-induced endothelial differentiation of BMSCs, pCMV6-CD163 or siRNA-CD163 was used to overexpress or knock down CD163 with $\mathrm{ABO}$ treatment, respectively. For knockdown of CD163, we detected the siRNA-CD163 efficiency in BMSCs by western blotting (Figures 3(a) and 3(b)). As shown in Figures 3(c) and $3(\mathrm{~d})$, on the basis of enhanced protein level of CD31 and Flk-1 after $50 \mu \mathrm{M}$ ABO treatment for $48 \mathrm{~h}$, pCMV6-CD163 further increased their protein level; it was more important that siRNA-CD163 significantly decreased their protein level. The data suggested that CD163 had an important role in ABOinduced endothelial differentiation of BMSCs.

3.3. CD163 Was Positively Regulated by Hmbox1 in BMSCs. To investigate the relationship between CD163 and Hmboxl in endothelial differentiation of BMSCs, we detected the protein level of CD163 and Hmboxl with $50 \mu \mathrm{M}$ ABO treatment for $48 \mathrm{~h}$ and transfected pCMV6-CD163 or siRNA-CD163 to BMSCs at the same time. ABO treatment significantly improved the protein level of CD163 (Figures 3(c) and 3(e)), whereas changed protein level of CD163 cannot influence the protein level of Hmboxl with ABO treatment (Figures 3(c) and 3(d)). And even without $\mathrm{ABO}$ treatment, transfected pCMV6-CD163 or siRNA-CD163 also cannot alter the protein level of Hmboxl (Figures 4(a) and 4(b)). According to our previous work, we deduced that Hmboxl was located upstream CD163 in this differentiation regulation pathway. We examined the protein level of CD163 after BMSCs were transfected with pCMV6-Hmboxl or siRNAHmboxl (Figures 4(c), 4(d), and 4(e)). Overexpression of Hmboxl upregulated the protein level of CD163, whereas knockdown of Hmboxl downregulated CD163 in BMSCs. These results confirmed that Hmboxl positively regulated CD163 in BMSCs.

3.4. CD163 Acted Upstream FGF-2 in the Differentiation of BMSCs into a VEC Lineage. Our previous study showed that $\mathrm{ABO}$ could induce mESC differentiation into VECs by enhancing Hmboxl expression and further upregulating the protein level of the important proangiogenic factor, FGF-2 [10]. To further understand the mechanism of CD163 in the differentiation of BMSCs to a VEC lineage, we detected the protein level of FGF-2 in BMSCs after being transfected with pCMV6-CD163 or pCMV6 empty vector for $48 \mathrm{~h}$. As shown in Figures 5(a) and 5(b), compared with the control group, the protein level of FGF-2 was significantly increased in BMSCs after CD163 overexpression. These data suggested that CD163 promoted the differentiation of BMSCs into a VEC lineage through enhancing FGF-2 signaling.

\section{Discussion}

In this study, we first studied the role of CD163 in positive regulation of the differentiation of BMSC into vascular endothelial-like cells. We demonstrated as well that CD163 took part in and further promoted the endothelial differentiation process induced by $\mathrm{ABO}$ in BMSCs. Moreover, overexpression of Hmboxl upregulated the protein level of CD163; meanwhile overexpression of CD163 upregulated the protein level of FGF-2. These data suggested that CD163 was involved in Hmbox1/CD163/FGF-2 signal pathway in the differentiation of BMSCs into vascular endothelial-like cells 
(Figure 5(c)). Collectively, the novel finding of this study was the role of CD163 in BMSC differentiation towards a VEC lineage. Meanwhile, we demonstrated that CD163 regulated the differentiation of BMSCs into vascular endothelial-like cells via Hmboxl/CD163/FGF-2 signaling (Figure 5(c)).

The hemoglobin $(\mathrm{Hb})$ scavenger receptor, $\mathrm{CD} 163$, has a key role in the control of inflammatory processes by the induction of anti-inflammatory pathways [33]. A recent report showed that CD163 expression was associated with angiogenesis [23]; however, little is known about its role in angiogenesis. It is for the first time proposed that CD163 plays an important role in the differentiation of BMSCs into vascular endothelial-like cells. The findings of this work provided a novel target for control of BMSC differentiation into a vascular endothelial lineage and for further investigation of CD163 functions.

It is very complex that the expression of CD163 is regulated by a variety of factors. On its promoter sequence, there are one major transcription start site, six alternative initiation sites, and possible binding sites for transcription factors like glucocorticoid receptor, PU.1, C/EBP, Ets-2, and AP-1, which have been shown to play an important role in myeloidspecific gene expression [34]. There is no TATA box in its proximal sequence, which also is a common feature of many myeloid-specific promoter sequences $[17,34]$. Hence, CD163 not only plays pivotal roles in monocytes and macrophages, but also may play important roles in BMSCs.

Hmboxl was a key factor in BMSC/mESC differentiation into VECs and upregulated Ets-1 in BMSC differentiation into VECs $[2,10]$. Here, we found that Hmboxl positively regulated CD163, while CD163 might be regulated by Ets- 2 [34]. Meanwhile, plenty of reports showed that Ets-1 and Ets2 are functionally redundant in lots of cases and required for endothelial cell survival [35-38]. It is a possible pathway that CD163 is positively regulated by Hmboxl/Ets-1 signaling, and, in our further investigation, we will verify this pathway of Hmbox1/Ets-1/CD163 signaling.

In summary, our data suggested that CD163, which was positively regulated by Hmboxl and acted upstream FGF-2, was a key regulator in this differentiation process. These findings provided a novel target for further investigating the gene control of BMSC differentiation into vascular endothelial-like cells.

\section{Conflict of Interests}

The authors declare that there is no conflict of interests regarding the publication of this paper.

\section{Acknowledgments}

This work was financially supported by Specialized Research Fund for the Doctoral Program of Higher Education (no. 20120131130010), the Science and Technology Development Project of Shandong Province (2014GSF118158 and 2015GSF121010), China Postdoctoral Science Foundation (nos. 201104637, 201003638, 20080441147, and 200802024), Shandong Excellent Young Scientist Award Fund (no.
BS2013SW001), and the Fundamental Research Funds of Shandong University (2014QY003).

\section{References}

[1] A. Forte, M. Finicelli, M. Mattia et al., "Mesenchymal stem cells effectively reduce surgically induced stenosis in rat carotids," Journal of Cellular Physiology, vol. 217, no. 3, pp. 789-799, 2008.

[2] L. Su, H. Zhao, C. Sun et al., "Role of Hmboxl in endothelial differentiation of bone-marrow stromal cells by a small molecule," ACS Chemical Biology, vol. 5, no. 11, pp. 1035-1043, 2010.

[3] J. Shao, C. Sun, L. Su, J. Zhao, S. Zhang, and J. Miao, "Phosphatidylcholine-specific phospholipase C/heat shock protein 70 (Hsp70)/transcription factor B-cell translocation gene 2 signaling in rat bone marrow stromal cell differentiation to cholinergic neuron-like cells," International Journal of Biochemistry \& Cell Biology, vol. 44, no. 12, pp. 2253-2260, 2012.

[4] S. Raeth, B. Sacchetti, G. Siegel et al., "A mouse bone marrow stromal cell line with skeletal stem cell characteristics to study osteogenesis in vitro and in vivo," Stem Cells and Development, vol. 23, no. 10, pp. 1097-1108, 2014.

[5] T. J. Poole, E. B. Finkelstein, and C. M. Cox, "The role of FGF and VEGF in angioblast induction and migration during vascular development," Developmental Dynamics, vol. 220, no. 1, pp. 117, 2001.

[6] W.-M. Yue, W. Liu, Y.-W. Bi et al., "Mesenchymal stem cells differentiate into an endothelial phenotype, reduce neointimal formation, and enhance endothelial function in a rat vein grafting model," Stem Cells and Development, vol. 17, no. 4, pp. 785-793, 2008.

[7] R. D. S. Nandoe, A. Hurtado, A. D. O. Levi, A. Grotenhuis, and M. Oudega, "Bone marrow stromal cells for repair of the spinal cord: towards clinical application," Cell Transplantation, vol. 15, no. 7, pp. 563-577, 2006.

[8] W. Fan, R. Crawford, and Y. Xiao, "The ratio of VEGF/PEDF expression in bone marrow mesenchymal stem cells regulates neovascularization," Differentiation, vol. 81, no. 3, pp. 181-191, 2011.

[9] J. Xu, X. Liu, J. Chen et al., "Simvastatin enhances bone marrow stromal cell differentiation into endothelial cells via notch signaling pathway," American Journal of Physiology-Cell Physiology, vol. 296, no. 3, pp. C535-C543, 2009.

[10] L. Han, J. Shao, L. Su et al., "A chemical small molecule induces mouse embryonic stem cell differentiation into functional vascular endothelial cells via Hmboxl," Stem Cells and Development, vol. 21, no. 15, pp. 2762-2769, 2012.

[11] G. Zwadlo, R. Voegeli, K. Schulze Osthoff, and C. Sorg, "A monoclonal antibody to a novel differentiation antigen on human macrophages associated with the down-regulatory phase of the inflammatory process," Experimental Cell Biology, vol. 55, no. 6, pp. 295-304, 1987.

[12] T. Kishimoto, S. Goyert, H. Kikutani et al., "CD antigens 1996," Blood, vol. 89, no. 10, p. 3502, 1997.

[13] M. M. Van den Heuvel, C. P. Tensen, J. H. Van As et al., "Regulation of CD 163 on human macrophages: cross-linking of CD163 induces signaling and activation," Journal of Leukocyte Biology, vol. 66, no. 5, pp. 858-866, 1999.

[14] J. H. Graversen, M. Madsen, and S. K. Moestrup, "CD163: a signal receptor scavenging haptoglobin-hemoglobin complexes from plasma," International Journal of Biochemistry and Cell Biology, vol. 34, no. 4, pp. 309-314, 2002. 
[15] M. B. Maniecki, A. Etzerodt, B. P. Ulhøi et al., "Tumorpromoting macrophages induce the expression of the macrophage-specific receptor CD163 in malignant cells," International Journal of Cancer, vol. 131, no. 10, pp. 2320-2331, 2012.

[16] C. Urbich, A. I. De Souza, L. Rossig et al., "Proteomic characterization of human early pro-angiogenic cells," Journal of Molecular and Cellular Cardiology, vol. 50, no. 2, pp. 333-336, 2011.

[17] P. Akila, V. Prashant, M. N. Suma, S. N. Prashant, and T. R. Chaitra, "CD163 and its expanding functional repertoire," Clinica Chimica Acta, vol. 413, no. 7-8, pp. 669-674, 2012.

[18] J. Guetta, M. Strauss, N. S. Levy, L. Fahoum, and A. P. Levy, "Haptoglobin genotype modulates the balance of Th1/Th2 cytokines produced by macrophages exposed to free hemoglobin," Atherosclerosis, vol. 191, no. 1, pp. 48-53, 2007.

[19] L. C. Bover, M. Cardó-Vila, A. Kuniyasu et al., "A previously unrecognized protein-protein interaction between TWEAK and CD163: potential biological implications," Journal of Immunology, vol. 178, no. 12, pp. 8183-8194, 2007.

[20] M. J. Kaplan, E. E. Lewis, E. A. Shelden et al., "The apoptotic ligands TRAIL, TWEAK, and fas ligand mediate monocyte death induced by autologous lupus T cells," Journal of Immunology, vol. 169, no. 10, pp. 6020-6029, 2002.

[21] S. R. Wiley, L. Cassiano, T. Lofton et al., "A novel TNF receptor family member binds TWEAK and is implicated in angiogenesis," Immunity, vol. 15, no. 5, pp. 837-846, 2001.

[22] T. C. Polek, M. Talpaz, B. G. Darnay, and T. Spivak-Kroizman, "TWEAK mediates signal transduction and differentiation of RAW264.7 cells in the absence of Fn14/TweakR. Evidence for a second TWEAK receptor," Journal of Biological Chemistry, vol. 278, no. 34, pp. 32317-32323, 2003.

[23] Y. W. Koh, C.-S. Park, D. H. Yoon, C. Suh, and J. Huh, "CD163 expression was associated with angiogenesis and shortened survival in patients with uniformly treated classical Hodgkin lymphoma," PLoS ONE, vol. 9, no. 1, Article ID e87066, 2014.

[24] M. F. Pittenger, A. M. Mackay, S. C. Beck et al., "Multilineage potential of adult human mesenchymal stem cells," Science, vol. 284, no. 5411, pp. 143-147, 1999.

[25] N. Wang, K. Xie, S. Huo, J. Zhao, S. Zhang, and J. Miao, "Suppressing phosphatidylcholine-specific phospholipase $\mathrm{C}$ and elevating ROS level, NADPH oxidase activity and Rb level induced neuronal differentiation in mesenchymal stem cells," Journal of Cellular Biochemistry, vol. 100, no. 6, pp. 1548-1557, 2007.

[26] Y. Kureishi, Z. Luo, I. Shiojima et al., "The HMG-CoA reductase inhibitor simvastatin activates the protein kinase Akt and promotes angiogenesis in normocholesterolemic animals," Nature Medicine, vol. 6, no. 9, pp. 1004-1010, 2000.

[27] X. Lv, L. Su, D. Yin et al., "Knockdown of integrin $\beta 4$ in primary cultured mouse neurons blocks survival and induces apoptosis by elevating NADPH oxidase activity and reactive oxygen species level," International Journal of Biochemistry and Cell Biology, vol. 40, no. 4, pp. 689-699, 2008.

[28] N. Wang, C. Sun, S. Huo et al., "Cooperation of phosphatidylcholine-specific phospholipase $\mathrm{C}$ and basic fibroblast growth factor in the neural differentiation of mesenchymal stem cells in vitro," International Journal of Biochemistry and Cell Biology, vol. 40, no. 2, pp. 294-306, 2008.

[29] A.-Y. Du, B.-X. Zhao, D.-L. Yin, S.-L. Zhang, and J.-Y. Miao, "Discovery of a novel small molecule, 1-ethoxy-3-(3,4methylenedioxyphenyl)-2-propanol, that induces apoptosis in
A549 human lung cancer cells," Bioorganic and Medicinal Chemistry, vol. 13, no. 13, pp. 4176-4183, 2005.

[30] T. Asahara, T. Murohara, A. Sullivan et al., "Isolation of putative progenitor endothelial cells for angiogenesis," Science, vol. 275, no. 5302, pp. 964-967, 1997.

[31] J. Folkman, "Angiogenesis in cancer, vascular, rheumatoid and other disease," Nature Medicine, vol. 1, no. 1, pp. 27-31, 1995.

[32] J. D. Murray, "On the mechanochemical theory of biological pattern formation with application to vasculogenesis," Comptes Rendus Biologies, vol. 326, no. 2, pp. 239-252, 2003.

[33] A. Etzerodt and S. K. Moestrup, "CD163 and inflammation: biological, diagnostic, and therapeutic aspects," Antioxidants and Redox Signaling, vol. 18, no. 17, pp. 2352-2363, 2013.

[34] M. Ritter, C. Buechler, T. Langmann, E. Orso, J. Klucken, and G. Schmitz, "The scavenger receptor CD163: regulation, promoter structure and genomic organization," Pathobiology, vol. 67, no. 5-6, pp. 257-261, 2000.

[35] I. G. Maroulakou and D. B. Bowe, "Expression and function of Ets transcription factors in mammalian development: a regulatory network," Oncogene, vol. 19, no. 55, pp. 6432-6442, 2000.

[36] G. Wei, R. Srinivasan, C. Z. Cantemir-Stone et al., "Ets1 and Ets2 are required for endothelial cell survival during embryonic angiogenesis," Blood, vol. 114, no. 5, pp. 1123-1130, 2009.

[37] P. Oettgen, "Functional redundancy of Ets1 and Ets2," Blood, vol. 114, no. 5, pp. 934-935, 2009.

[38] P. Vivekanand and I. Rebay, "The SAM domain of human TEL2 can abrogate transcriptional output from TEL1 (ETV-6) and ETS1/ETS2," PLoS ONE, vol. 7, no. 5, Article ID e37151, 2012. 

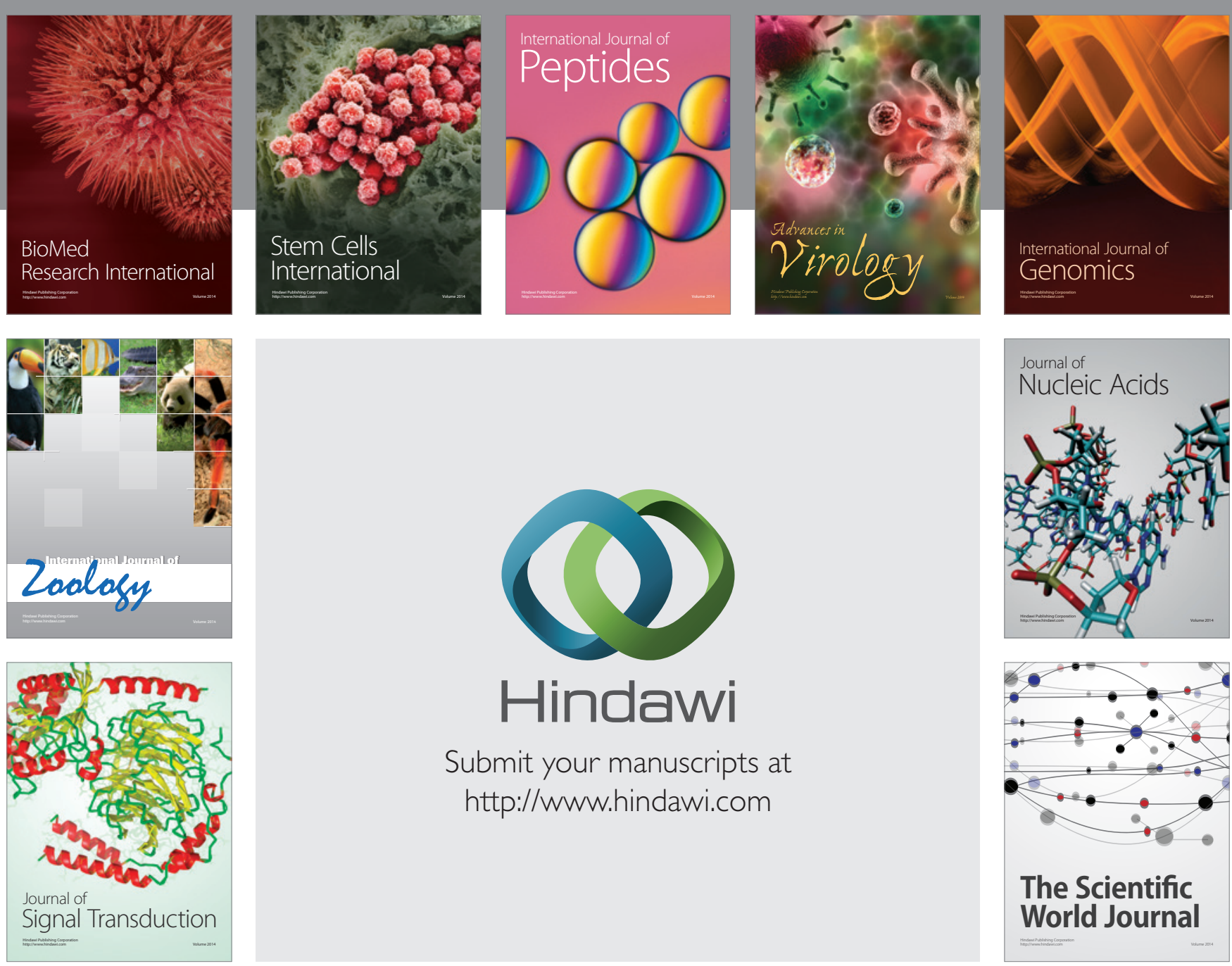

Submit your manuscripts at

http://www.hindawi.com
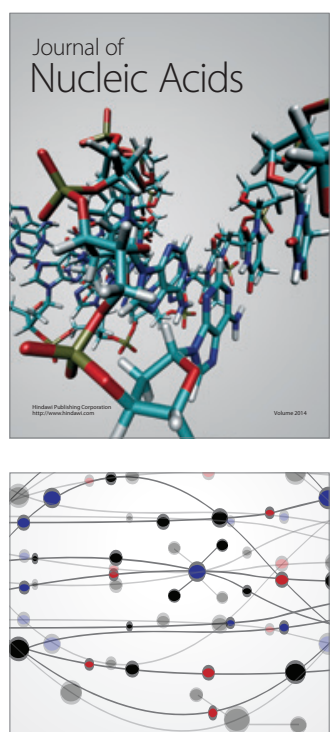

The Scientific World Journal
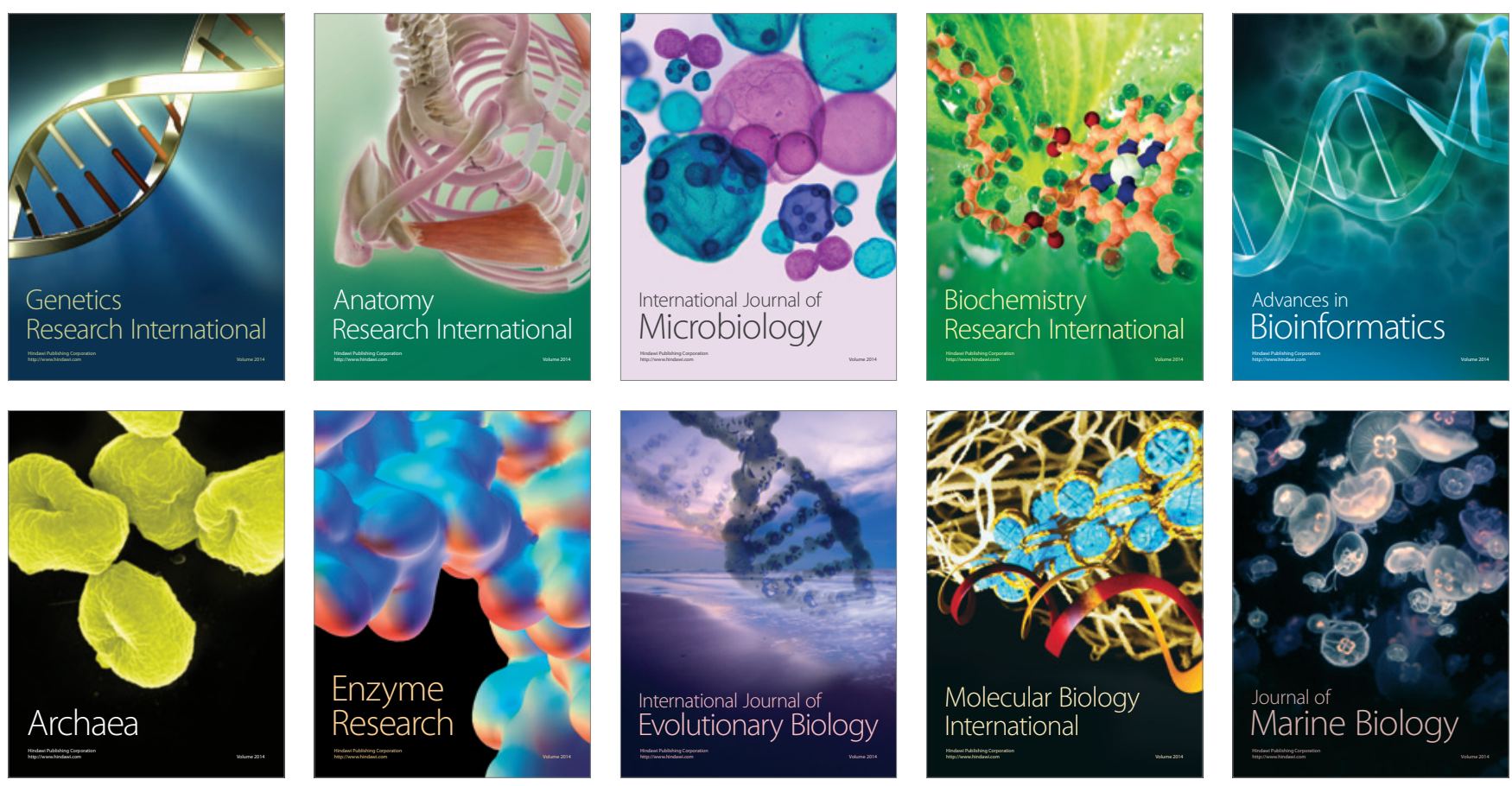